\title{
A Study of the Anisotropic Static Elasticity System in Thin Domain
}

\author{
Yassine Letoufa, ${ }^{1}$ Salah Mahmoud Boulaaras $\mathbb{D},{ }^{2,3}$ Hamid Benseridi, ${ }^{4}$ Mourad Dilmi, ${ }^{4}$ \\ and Asma Alharbi $\mathbb{D D}^{2}$ \\ ${ }^{1}$ Department of Mathematics and Computer Sciences, University of El Oued, El Oued, PO Box 789 39000, Algeria \\ ${ }^{2}$ Department of Mathematics, College of Sciences and Arts, ArRass, Qassim University, Saudi Arabia \\ ${ }^{3}$ Laboratory of Fundamental and Applied Mathematics of Oran (LMFAO), University of Oran 1, Oran, 31000 Oran, Algeria \\ ${ }^{4}$ Applied Mathematics Laboratory, Department of Mathematics, Faculty of Sciences, University of Ferhat ABBAS-Sétif 1, \\ 19000, Algeria
}

Correspondence should be addressed to Salah Mahmoud Boulaaras; s.boularas@qu.edu.sa

Received 7 June 2021; Accepted 16 July 2021; Published 31 July 2021

Academic Editor: Fanglei Wang

Copyright ( 2021 Yassine Letoufa et al. This is an open access article distributed under the Creative Commons Attribution License, which permits unrestricted use, distribution, and reproduction in any medium, provided the original work is properly cited.

We study the asymptotic behavior of solutions of the anisotropic heterogeneous linearized elasticity system in thin domain of $\mathbb{R}^{3}$ which has a fixed cross-section in the $\mathbb{R}^{2}$ plane with Tresca friction condition. The novelty here is that stress tensor has given by the most general form of Hooke's law for anisotropic materials. We prove the convergence theorems for the transition 3D-2D when one dimension of the domain tends to zero. The necessary mathematical framework and (2D) equation model with a specific weak form of the Reynolds equation are determined. Finally, the properties of solution of the limit problem are given, in which it is confirmed that the limit problem is well defined.

\section{Introduction}

In this paper, we are interested of the asymptotic behavior of the linear elasticity system in a domain of $\mathbb{R}^{3}$ with a Tresca friction condition where the boundary of this domain has a fixed cross-section in dimension 2 and a small thickness. One of the objectives of this study is to obtain twodimensional equation that allows a reasonable description of the phenomenon occurring in the three-dimensional domain by passing the limit to 0 on the small thickness of the domain (3D). Let us mention for example [1-8] in which the authors worked on the asymptotic behavior for the linearized elasticity system with different boundary conditions. Some problems of Newtonian or non-Newtonian fluids are considered in [9-11] where the authors proved a limit problem that gives a distribution of velocity and pressure through the weak form of the Reynolds equation. In $[6,7]$, the authors demonstrate the transition 3D-1D in anisotropic heterogeneous linearized elasticity; so, we mention here that this phenomenon has been studied only about strong solutions, without friction law. Benseridi in [2] investigated the asymptotic analysis of a dynamical problem of linear elasticity with
Tresca's friction. The static case with a nonlinear term for linear elastic materials has been considered in [3]. See another situation in [4] where the paper concerns asymptotic derivation of frictionless contact models for elastic rods on a foundation with normal compliance. Recently, the authors in [5, 12] have proved the asymptotic behavior of a frictionless contact problem between two elastic bodies, when the vertical dimension of the two domain reaches zero. However, all these papers have been only restricted in a homogeneous and isotropic case of elastic materials.

The present work is a follow-up of $[2,3,5]$ to study the heterogeneous and anisotropic situation with Tresca's friction. Here, the stress tensor with its components is given by the generalized Hooke's law (see [13]): $\sigma^{\varepsilon}=A^{\varepsilon} e\left(u^{\varepsilon}\right)$, where $u^{\varepsilon}$ denotes the displacement vector, $e\left(u^{\varepsilon}\right)$ is the linearized strain tensor, and $A^{\varepsilon}$ is the fourth order tensor which describes the elastic properties of the material. Many materials that follow the linear elastic model, although they are well made, are not subject to the assumptions of isotropy, for example, wood, reinforced concrete, composite materials, and many biological materials, where the mechanical properties of these materials differ according to the directions of space; in that case, the elasticity 
operator depends on the location of the point (see $[14,15])$. Necas in [7] and Sofonea in [16] established the existence of a weak solution for the static frictional contact problem involving linearly elastic and viscoelastic materials, by using a results of convex optimization [17], and numerical approximation of this problem was studied in [18]. For the variational analysis of various contact problems, we mention excellent references in $[14,15]$. Mathematically, the asymptotic analysis is more difficult since in general, the limit problem involves an equation that takes into account the anisotropy of the medium, and it is thus important to identify the elastic components of $A^{\varepsilon}$ that appear in the (2D) equation model.

The paper is organized as follows; in section 2, the strong and weak formulation of the problem is given in terms of $u^{\varepsilon}$ and also the related existence and uniqueness of the weak solution. In section 3, we introduce a scaling, and we find some estimates on the displacement which are independent of the parameter $\varepsilon$. In section 4 , we state the main results concerning the existence of a weak limit $u^{*}$ of $u^{\varepsilon}$, the (2D) equation model with a specific weak form of the Reynolds equation is proved, the limit form of the Tresca boundary conditions is formulated, and finally, the uniqueness of $u^{*}$ is given.

\section{Mathematical Formulation}

Let $\omega$ be an open set in $\mathbb{R}$ with Lipschitz boundary, and we consider a smooth function $h: \omega \longrightarrow \mathbb{R}$ be a class $C^{1}$ such that $0<h_{\min } \leq h(x) \leq h_{\max }$, for all $x \in \omega$, where $h_{\min }$ and $h_{\text {max }}$ are constants. We define the smooth bounded domain $\Omega$ whose boundary has a flat part $\omega$,

$$
\Omega=\left\{(x, z) \in \mathbb{R}^{3}, x \in \omega, 0<z<h(x)\right\} .
$$

We denote by $\Gamma_{1}$ is the upper boundary of the equation $z=h(x)$, and $\Gamma_{L}$ is the lateral boundary.

Let $\varepsilon>0$ be a small parameter, and we define $\Omega^{\varepsilon}$ be the change of scale $z=x_{3} / \varepsilon$ and the points of $\Omega$,

$$
\Omega^{\varepsilon}=\left\{\left(x, x_{3}\right) \in \mathbb{R}^{3}, x \in \omega, 0<x_{3}<\operatorname{ch}(x)\right\} .
$$

We have $\Gamma^{\varepsilon}=\bar{\omega} \cup \bar{\Gamma}_{1}^{\varepsilon} \cup \bar{\Gamma}_{L}^{\varepsilon}$ which its boundary of $\Omega^{\varepsilon}$ and where $\Gamma_{1}^{\varepsilon}$ is the upper surface defined by $x_{3}=\operatorname{ch}(x)$, and $\Gamma_{L}^{\varepsilon}$ is the lateral boundary. The unit outward normal to $\Gamma^{\varepsilon}$ is denoted by $v$. It follows that there is correspondence between the functions $\phi: \Omega^{\varepsilon} \longrightarrow \mathbb{R}^{n}$ and $\widehat{\phi}: \Omega \longrightarrow \mathbb{R}^{n}(n=1,2,3)$ given by $\widehat{\phi}(x, z)=\phi\left(x, x_{3}\right)$.

Let $H^{1 / 2}(\Gamma)^{3}$ be the space of traces of functions on $\Gamma$ of functions from $H^{1}(\Omega)^{3}$, and we use the vector function $g \in$ $H^{1 / 2}(\Gamma)^{3}$ such that

$$
\int_{\Gamma} g \cdot v d s=0
$$

We denote by $\mathbb{S}_{n}$ the space of symmetric tensors on $\mathbb{R}^{n}$ and $|$.$| the Euclidean norm on \mathbb{R}^{n}$ and $\mathbb{S}_{n}$. Here and below, the indices $i, j, k, l$ run between 1 and 3 , and the summation convention overrepeated indices is adopted.
The basic equations of frictionless contact problem for the anisotropic heterogeneous elastic body occupy the domain $\Omega^{\varepsilon}$ as follows:

The equations of equilibrium are as follows:

$$
\begin{aligned}
\frac{\partial \sigma_{i j}^{\varepsilon}}{\partial x_{j}}+f_{i}^{\varepsilon} & =0 \operatorname{in} \Omega^{\varepsilon}, \\
\sigma_{i j}^{\varepsilon}\left(u^{\varepsilon}\right) & =A_{i j k l}^{\varepsilon} e_{k l}\left(u^{\varepsilon}\right) \operatorname{in} \Omega^{\varepsilon},
\end{aligned}
$$

where the vector $f^{\varepsilon}=\left(f_{1}^{\varepsilon}, f_{2}^{\varepsilon}, f_{3}^{\varepsilon}\right)$ represents the forces of density, $u^{\varepsilon}=\left(u_{1}^{\varepsilon}, u_{2}^{\varepsilon}, u_{3}^{\varepsilon}\right)$ is the displacement field, the elements $A_{i j k l}^{\varepsilon}$ denote the components of elasticity tensor $A^{\varepsilon}$, and $e_{i j}\left(u^{\varepsilon}\right)$ is the rate of deformation operator,

$$
e_{i j}\left(u^{\varepsilon}\right)=\left(e\left(u^{\varepsilon}\right)\right)_{i j}=\frac{1}{2}\left(\frac{\partial u_{i}^{\varepsilon}}{\partial x_{j}}+\frac{\partial u_{j}^{\varepsilon}}{\partial x_{i}}\right) .
$$

On $\Gamma_{L}^{\varepsilon}$, the displacement is known:

$$
u^{\varepsilon}=g^{\varepsilon} \text { on } \Gamma_{L}^{\varepsilon}
$$

On $\Gamma_{1}^{\varepsilon}$, we assume that the elastic body is held fixed:

$$
u^{\varepsilon}=g^{\varepsilon}=0 \text { on } \Gamma_{1}^{\mathcal{E}}
$$

On the surface $\omega$, we assume that the contact is bilateral:

$$
u^{\varepsilon} \cdot v=g^{\varepsilon} \cdot v=0
$$

and satisfies the Tresca boundary condition [7] with friction function $k^{\varepsilon}$;

$$
\left\{\begin{array}{l}
\left|\sigma_{\tau}^{\varepsilon}\right|<k^{\varepsilon} u_{\tau}^{\varepsilon}=s, \\
\left|\sigma_{\tau}^{\varepsilon}\right|=k^{\varepsilon} \exists \lambda \geq 0 \text { suchthat } u_{\tau}^{\varepsilon}=s-\lambda \sigma_{\tau}^{\varepsilon},
\end{array}\right.
$$

where $s=g^{\varepsilon}$ on $\omega . u_{\tau}^{\varepsilon}, \sigma_{\tau}^{\varepsilon}$, and $\sigma_{v}^{\varepsilon}$ are the tangential displacement, the tangential, and the normal stress tensor, respectively, with

$$
u_{\tau_{i}}^{\varepsilon}=u_{i}^{\varepsilon}-u_{j}^{\varepsilon} v_{j} v_{i}, \sigma_{\tau_{i}}^{\varepsilon}=\sigma_{i j}^{\varepsilon} \cdot v_{j}-\left(\sigma_{v}^{\varepsilon}\right) \cdot v_{i}, \sigma_{v}^{\varepsilon}=\left(\sigma^{\varepsilon} \cdot v\right) \cdot v
$$

Consider now the following closed convex subset of $H^{1}$ $\left(\Omega^{\varepsilon}\right)^{3}$ given by

$$
K^{\varepsilon}=\left\{\phi \in H^{1}\left(\Omega^{\varepsilon}\right)^{3}: \phi=g^{\varepsilon} \text { on } \Gamma_{1}^{\varepsilon} \cup \Gamma_{L}^{\varepsilon}, \phi \cdot v=0 \text { on } \omega\right\} .
$$

Let us introduce the form $a: K^{\varepsilon} \times K^{\varepsilon} \longrightarrow \mathbb{R}$ and the functional $J^{\varepsilon}: K^{\varepsilon} \longrightarrow \mathbb{R}^{+}$defined by

$$
\begin{aligned}
a\left(u^{\varepsilon}, \phi\right) & =\int_{\Omega^{\varepsilon}} A_{i j k l}^{\varepsilon} e_{k l}\left(u^{\varepsilon}\right) e_{i j}(\phi) d x d x_{3}, \\
J^{\varepsilon}(\phi) & =\int_{\omega} k^{\varepsilon}|\phi-s| d x
\end{aligned}
$$


In the study of the mechanical problem (3)-(10), we assume that all components $A_{i j k l}^{\varepsilon}$ belong to $L^{\infty}\left(\Omega^{\varepsilon}\right)$ and satisfy the usual properties of symmetry and ellipticity [19], i.e.,

$$
A_{i j k l}^{\varepsilon}=A_{j i k l}^{\varepsilon}=A_{k l i j}^{\varepsilon} \in L^{\infty}\left(\Omega^{\varepsilon}\right),
$$

and there exists a constant $\mu>0$ such that

$$
A_{i j k l}^{\varepsilon}(y) \xi_{k l} \xi_{i j} \geq \mu|\xi|^{2} \forall \xi \in \mathbb{S}_{3} \text {, a.e. } y \in \Omega^{\varepsilon} .
$$

Remark 1. It follows from previous properties and by Korn's inequality (see [16], pp. 79), that the bilinear form $a$ is coercive and continuous, i.e.,

$$
\begin{gathered}
a(\phi, \phi) \geq \mu C_{K}\|\nabla \phi\|_{L^{2}\left(\Omega^{\varepsilon}\right)}^{2} \forall \phi \in K^{\varepsilon}, \\
|a(\varphi, \psi)| \leq M\|\nabla \phi\|_{L^{2}\left(\Omega^{\varepsilon}\right)}\|\nabla \psi\|_{L^{2}\left(\Omega^{\varepsilon}\right)} \forall \phi, \psi \in K^{\varepsilon},
\end{gathered}
$$

where $M=\max _{1 \leq i, j, k, l \leq 3}\left\|A_{i j k l}^{\varepsilon}\right\|_{L^{\infty}\left(\Omega^{\varepsilon}\right)}$ and $C_{K}$ denoting a positive constant depends on $\Omega^{\varepsilon}, \Gamma_{1}^{\varepsilon}$, and $\Gamma_{L}^{\varepsilon}$.

Lemma 2. Assuming that $f^{\varepsilon} \in L^{2}\left(\Omega^{\varepsilon}\right)^{3}$ and $k^{\varepsilon} \in L^{\infty}(\omega)$, the variational formulation of problem (3)-(10) is equivalent to

Find $u^{\varepsilon} \in K^{\varepsilon}$ satisfying

$a\left(u^{\varepsilon}, \phi-u^{\varepsilon}\right)+J^{\varepsilon}(\phi)-J^{\varepsilon}\left(u^{\varepsilon}\right) \geq \int_{\Omega^{\varepsilon}} f^{\varepsilon} \cdot\left(\phi-u^{\varepsilon}\right) d x d x_{3} \forall \phi \in K^{\varepsilon}$,

for every \& small fixed.

Moreover, if the assumptions of (14) and (15) hold, then the variational inequality (18) has a unique solution $u^{\varepsilon} \in K^{\varepsilon}$.

Remark 3. A problem of the form (18) is called an elliptic variational inequality of the second kind ([17]). The following theorem (see [19], Theorem 6) allows us to replace the variational inequality (18) by a minimization problem. Thus, we will not repeat the proof, but our goal is to study the asymptotic behavior.

\section{Some Estimates in Fixed Domain}

To be able to study the asymptotic behavior of the solutions of (18), we use the change of variable $z=x_{3} / \varepsilon$, to return to the fixed domain $\Omega$, and then we define the following functions in $\Omega$ :

$$
\widehat{u}_{i}^{\varepsilon}(x, z)=u_{i}^{\varepsilon}\left(x, x_{3}\right) \quad \text { for } i=1,2,3 .
$$

For the data $\widehat{A}_{i j k l}, \widehat{f}_{i}$, and $\widehat{k}$, we have the following relations:
$\widehat{A}_{i j k l}(x, z)=A_{i j k l}^{\varepsilon}\left(x, x_{3}\right), \quad \widehat{f}_{i}(x, z)=\varepsilon^{2} f_{i}^{\varepsilon}\left(x, x_{3}\right)$ and $\widehat{k}=\varepsilon k^{\varepsilon}$,

(for $1 \leq i, j, k, l \leq 3$ ).

Let

$$
\begin{aligned}
K & =\left\{v \in H^{1}(\Omega)^{3}: v=\operatorname{gon} \Gamma_{L} \cup \Gamma_{1}, v \cdot v=0 \text { on } \omega\right\}, \\
V_{z} & =\left\{v=\left(v_{1}, v_{2}, v_{3}\right) \in L^{2}(\Omega)^{3}: \frac{\partial v_{i}}{\partial z}\right. \\
& \left.\in L^{2}(\Omega), i=1,2,3 ; v=0 \mathrm{on} \Gamma_{1}\right\} .
\end{aligned}
$$

$V_{z}$ is a Banach space for the following norm:

$$
\|v\|_{V_{z}}=\left[\sum_{i=1}^{3}\left(\left\|v_{i}\right\|_{L^{2}(\Omega)}^{2}+\left\|\frac{\partial v_{i}}{\partial z}\right\|_{L^{2}(\Omega)}^{2}\right)\right]^{1 / 2} .
$$

Everywhere in the sequel, the indexes $\alpha, \beta, \gamma$ and $\delta$ run from 1 to 2 , and summation over repeated indices is implied. Follow the same steps as in $[6,12]$, passing to the fixed domain $\Omega$, and using the symmetry of $\sigma_{i j}^{\varepsilon}$ and $A_{i j k l}^{\varepsilon}$, after multiplication by $\varepsilon$, we have (18) that is equivalent to

Find $\widehat{u}^{\varepsilon} \in K$, such that

$$
\begin{gathered}
\widehat{a}\left(\widehat{u}^{\varepsilon}, \widehat{\phi}-\widehat{u}^{\varepsilon}\right)+\int_{\omega} \widehat{k}|\widehat{\phi}-s| d x-\int_{\omega} \widehat{k}\left|\widehat{u}^{\varepsilon}-s\right| d x \\
\geq \sum_{i=1}^{3} \int_{\Omega} \widehat{f}_{i}\left(\widehat{\phi}_{i}-\widehat{u}_{i}^{\varepsilon}\right) d x d z, \forall \widehat{\phi} \in K,
\end{gathered}
$$

where

$$
\begin{aligned}
\widehat{a}\left(\widehat{u}^{\varepsilon}, \widehat{\phi}\right)= & \varepsilon^{2} \int_{\Omega} \widehat{A}_{\alpha \beta \gamma \theta} \widehat{e}_{\gamma \theta}\left(\widehat{u}^{\varepsilon}\right) \frac{\partial \widehat{\phi}_{\alpha}}{\partial x_{\beta}} d x d z \\
& +2 \varepsilon \int_{\Omega} \widehat{A}_{\alpha 3 \gamma \theta} \widehat{e}_{\gamma \theta}\left(\widehat{u}^{\varepsilon}\right) \frac{\partial \widehat{\phi}_{\alpha}}{\partial z} d x d z \\
& +2 \varepsilon^{2} \int_{\Omega} \widehat{A}_{\alpha \beta \gamma 3} \widehat{e}_{\gamma 3}\left(\widehat{u}^{\varepsilon}\right) \frac{\partial \widehat{\phi}_{\alpha}}{\partial x_{\beta}} d x d z \\
& +4 \varepsilon \int_{\Omega} \widehat{A}_{\alpha 3 \gamma 3} \widehat{e}_{\gamma 3}\left(\widehat{u}^{\varepsilon}\right) \frac{\partial \widehat{\phi}_{\alpha}}{\partial z} d x d z \\
& +\varepsilon^{2} \int_{\Omega} \widehat{A}_{\alpha \beta 33} \widehat{e}_{33}\left(\widehat{u}^{\varepsilon}\right) \frac{\partial \widehat{\phi}_{\alpha}}{\partial x_{\beta}} d x d z \\
& +\varepsilon \int_{\Omega} \widehat{A}_{33 \alpha \beta} \widehat{e}_{\alpha \beta}\left(\widehat{u}^{\varepsilon}\right) \frac{\partial \widehat{\phi}_{3}}{\partial z} d x d z \\
& +2 \varepsilon \int_{\Omega} \widehat{A}_{\alpha 333} \widehat{e}_{33}\left(\widehat{u}^{\varepsilon}\right) \frac{\partial \widehat{\phi}_{\alpha}}{\partial z} d x d z \\
& +2 \varepsilon \int_{\Omega} \widehat{A}_{33 \alpha 3} \widehat{e}_{\alpha 3}\left(\widehat{u}^{\varepsilon}\right) \frac{\partial \widehat{\phi}_{3}}{\partial z} d x d z \\
& +\varepsilon \int_{\Omega} \widehat{A}_{3333} \widehat{e}_{33}\left(\widehat{u}^{\varepsilon}\right) \frac{\partial \widehat{\phi}_{3}}{\partial z} d x d z
\end{aligned}
$$


and $\widehat{e}\left(\widehat{u}^{\varepsilon}\right)=\left(\widehat{e}_{i j}\left(\widehat{u}^{\varepsilon}\right)\right)_{i j}$ is given by the relations

$$
\begin{array}{ll}
\widehat{e}_{i j}\left(\widehat{u}^{\varepsilon}\right)=\frac{1}{2}\left(\frac{\partial \widehat{u}_{i}^{\varepsilon}}{\partial x_{j}}+\frac{\partial \widehat{u}_{j}^{\varepsilon}}{\partial x_{i}}\right), & i, j=1,2, \\
\widehat{e}_{i 3}\left(\widehat{u}^{\varepsilon}\right)=\widehat{e}_{3 i}\left(\widehat{u}^{\varepsilon}\right)=\frac{1}{2}\left(\frac{1}{\varepsilon} \frac{\partial \widehat{u}_{i}^{\varepsilon}}{\partial z}+\frac{\partial \widehat{u}_{3}^{\varepsilon}}{\partial x_{i}}\right), & i=1,2 . \\
\widehat{e}_{33}\left(\widehat{u}^{\varepsilon}\right)=\frac{1}{\varepsilon} \frac{\partial \widehat{u}_{3}^{\varepsilon}}{\partial z} &
\end{array}
$$

Lemma 4. Under the assumptions of Lemma 2, there exists a constant $C>0$ independent of $\varepsilon$, such that

$$
\sum_{i=1}^{3}\left\|\frac{\partial \widehat{u}_{i}^{\varepsilon}}{\partial z}\right\|_{L^{2}(\Omega)}^{2}+\varepsilon^{2} \sum_{i, j=1}^{2}\left\|\frac{\partial \widehat{u}_{i}^{\varepsilon}}{\partial x_{j}}\right\|_{L^{2}(\Omega)}^{2}+\varepsilon^{2} \sum_{j=1}^{2}\left\|\frac{\partial \widehat{u}_{3}^{\varepsilon}}{\partial x_{j}}\right\|_{L^{2}(\Omega)}^{2} \leq C .
$$

Proof. Assume that $u^{\varepsilon}$ is a solution of $(2.12)$. As $J^{\varepsilon}\left(u^{\varepsilon}\right) \geq 0$, then

$$
\begin{aligned}
a\left(u^{\varepsilon}, u^{\varepsilon}\right) \leq & a\left(u^{\varepsilon}, \phi\right)+J^{\varepsilon}(\phi)+\int_{\Omega^{\varepsilon}} f^{\varepsilon} \cdot u^{\varepsilon} d x d x_{3} \\
& -\int_{\Omega^{\varepsilon}} f^{\varepsilon} \cdot \phi d x d x_{3}, \quad \forall \phi \in K^{\varepsilon} .
\end{aligned}
$$

Using the Young's inequality

$$
a b \leq \eta^{2} \frac{a^{2}}{2}+\eta^{-2} \frac{b^{2}}{2}
$$

in (17) for $\eta=\sqrt{\mu C_{K} / 2}$, we find

$$
\left|a\left(u^{\varepsilon}, \phi\right)\right| \leq \frac{\mu C_{K}}{4}\left\|\nabla u^{\varepsilon}\right\|_{L^{2}\left(\Omega^{\varepsilon}\right)}^{2}+\frac{M}{\mu C_{K}}\|\nabla \phi\|_{L^{2}\left(\Omega^{\varepsilon}\right)}^{2} .
$$
we get

Also, by the Cauchy-Schwarz and Poincarés inequalities,

$$
\left|\int_{\Omega^{\varepsilon}} f^{\varepsilon} \cdot \phi d x d x_{3}\right| \leq \varepsilon h_{\max }\left\|f^{\varepsilon}\right\|_{L^{2}\left(\Omega^{\varepsilon}\right)}\|\nabla \phi\|_{L^{2}\left(\Omega^{\varepsilon}\right)},
$$

then using Young's inequality for $\eta=\sqrt{1 / \mu C_{K}}$ to obtain

$$
\left|\int_{\Omega^{\varepsilon}} f^{\varepsilon} \cdot u^{\varepsilon} d x d x_{3}\right| \leq \frac{\left(\varepsilon h_{\max }\right)^{2}}{2 \mu C_{K}}\left\|f^{\varepsilon}\right\|_{L^{2}\left(\Omega^{\varepsilon}\right)}^{2}+\frac{\mu C_{K}}{2}\|\nabla \phi\|_{L^{2}\left(\Omega^{\varepsilon}\right)}^{2} .
$$

Using (16), (29), and (31) in (27), we get

$$
\begin{aligned}
\frac{\mu C_{K}}{4}\left\|\nabla u^{\varepsilon}\right\|_{L^{2}\left(\Omega^{\varepsilon}\right)}^{2} \leq & \frac{M}{\mu C_{K}}\|\nabla \phi\|_{L^{2}\left(\Omega^{\varepsilon}\right)}^{2}+\frac{\left(\varepsilon h_{\max }\right)^{2}}{\mu C_{K}}\left\|f^{\varepsilon}\right\|_{L^{2}\left(\Omega^{\varepsilon}\right)}^{2} \\
& +\frac{\mu C_{K}}{2}\|\nabla \phi\|_{L^{2}\left(\Omega^{\varepsilon}\right)}^{2}+\int_{\omega} k^{\varepsilon}|\phi-s| d x .
\end{aligned}
$$

Taking into account the $g$ function introduced in (5) and using [20] (lemma 2 pp.24), there exists a function $\tilde{g} \in H^{1}$ $(\Omega)^{3}$ such that

$$
\tilde{g}=\operatorname{gon} \Gamma_{L} \text { and } \quad \tilde{g} \cdot v=0 \text { on } \omega \cup \Gamma_{1} .
$$

Thus, choosing $\widehat{\phi}=\tilde{g}$ in (3.6), then multiplying product inequality by $\varepsilon$, and the fact that $g=s$ on $\omega$, we obtain

$$
\begin{aligned}
\frac{\mu C_{K}}{4} \varepsilon\left\|\nabla u^{\varepsilon}\right\|_{L^{2}\left(\Omega^{\varepsilon}\right)}^{2} \leq & \frac{\left(h_{\max }\right)^{2}}{\mu C_{K}}\|\hat{f}\|_{L^{2}(\Omega)}^{2} \\
& +\left(\frac{M}{\mu C_{K}}+\frac{\mu C_{K}}{2}\right)\|\nabla \tilde{g}\|_{L^{2}(\Omega)}^{2} .
\end{aligned}
$$

From [6], we can see the constant Korn $C_{K}$ contained in Remark 1 does not depend on $\varepsilon$ and $\phi$, for $\varepsilon \in] 0,1]$; moreover, by changing the data of $A^{\varepsilon}$, remark that $\mu$ and $M$ are independent of $\varepsilon$. Therefore, passing to the fixed domain $\Omega$, we get

$$
\begin{aligned}
\varepsilon\left\|\nabla u^{\varepsilon}\right\|_{L^{2}\left(\Omega^{\varepsilon}\right)}^{2}= & \sum_{i=1}^{3}\left\|\frac{\partial \widehat{u}_{i}^{\varepsilon}}{\partial z}\right\|_{L^{2}(\Omega)}^{2}+\varepsilon^{2}\left(\sum_{i, j=1}^{2}\left\|\frac{\partial \widehat{u}_{i}^{\varepsilon}}{\partial x_{j}}\right\|_{L^{2}(\Omega)}^{2}\right. \\
& \left.+\sum_{j=1}^{2}\left\|\frac{\partial \widehat{u}_{3}^{\varepsilon}}{\partial x_{j}}\right\|_{L^{2}(\Omega)}^{2}\right) \leq C,
\end{aligned}
$$

with

$$
C=\frac{4}{\mu C_{K}}\left[\frac{\left(h_{\max }\right)^{2}}{\mu C_{K}}\|\hat{f}\|_{L^{2}(\Omega)}^{2}+\left(\frac{M}{\mu C_{K}}+\frac{\mu C_{K}}{2}\right)\|\nabla \tilde{g}\|_{L^{2}(\Omega)}^{2}\right] .
$$

Lemma 5. Under the assumptions of Lemma 4, there exists $u^{*}=\left(u_{1}^{*}, u_{2}^{*}, u_{3}^{*}\right) \in V_{z}$ such that

$$
\widehat{u}^{\varepsilon}-u^{*} \text { weaklyin } V_{z},
$$

$$
\begin{gathered}
\varepsilon \frac{\partial \widehat{u}_{i}^{\varepsilon}}{\partial x_{\alpha}} \rightarrow 0 \text { weaklyin } L^{2}(\Omega)(i=1,2,3 \text { and } \alpha=1,2), \\
\varepsilon \widehat{e}_{\alpha \beta}\left(\widehat{u}^{\varepsilon}\right) \rightarrow 0, \text { weaklyinL } L^{2}(\Omega)(\alpha, \beta=1,2), \\
\varepsilon \widehat{e}_{\gamma 3}\left(\widehat{u}^{\varepsilon}\right)-\frac{1}{2} \frac{\partial u_{\gamma}^{*}}{\partial z} \text { weaklyin }^{2}(\Omega)(\gamma=1,2), \\
\varepsilon \widehat{\varepsilon}_{33}\left(\widehat{u}^{\varepsilon}\right) \rightarrow \frac{\partial u_{3}^{*}}{\partial z} \text { weaklyin }^{2}(\Omega) .
\end{gathered}
$$

Proof. From (26), there exists a fixed constant $C>0$ such that

$$
\left\|\frac{\partial \widehat{u}_{i}^{\varepsilon}}{\partial z}\right\|_{L^{2}(\Omega)}^{2} \leq C, \quad \text { for } i=1,2,3 .
$$


Using Poincarés inequality in the domain $\Omega$

$$
\left\|\hat{u}_{i}^{\varepsilon}\right\|_{L^{2}(\Omega)} \leq h_{\max }\left\|\frac{\partial \widehat{u}_{i}^{\varepsilon}}{\partial z}\right\|_{L^{2}(\Omega)}, \quad \text { for } i=1,2,3,
$$

we deduce that $\widehat{u}^{\varepsilon}$ is bounded in $V_{z}$. From the last two estimates, there exists $u^{*}=\left(u_{1}^{*}, u_{2}^{*}, u_{3}^{*}\right) \in V_{z}$ and satisfies (37). From (26), we can extract a subsequence such that $\varepsilon\left(\partial \widehat{u}_{i}^{\varepsilon} / \partial\right.$ $\left.x_{\alpha}\right) \rightarrow \eta$ in $L^{2}(\Omega)$; on the other hand, from (37), we deduce (38). Also, (39)-(41) follow from (37) and (38).

\section{Limit Problem and Main Result}

At the limit $\varepsilon=0$, we give the satisfactory equations of $u^{*}$ and the properties of solution of the limit problem for the system (3)-(10).

Theorem 6. With the same assumptions as Lemma 5 , $u^{*}$ satisfies

$$
\begin{aligned}
& \left\langle A^{*} \frac{\partial u^{*}}{\partial z}, \frac{\partial}{\partial z}\left(\widehat{\phi}-u^{*}\right)\right\rangle_{L^{2}(\Omega)}+\int_{\omega} \widehat{k}\left(|\widehat{\phi}-s|-\left|u^{*}-s\right|\right) d x \\
& \quad \geq \int_{\Omega} \widehat{f} \cdot\left(\widehat{\phi}-u^{*}\right) d x d z, \quad \forall \widehat{\phi} \in K,
\end{aligned}
$$

where the symmetric matrix $A^{*}$ is given by

$$
A^{*}=\left(\begin{array}{ccc}
4 \widehat{A}_{1313} & 4 \widehat{A}_{1323} & 2 \widehat{A}_{1333} \\
4 \widehat{A}_{2313} & 4 \widehat{A}_{2323} & 2 \widehat{A}_{2333} \\
2 \widehat{A}_{3313} & 2 \widehat{A}_{3323} & \widehat{A}_{3333}
\end{array}\right) .
$$

Moreover, we have

$$
\left.\begin{array}{l}
-\frac{\partial}{\partial z}\left\{4 \widehat{A}_{\alpha 3 \gamma 3} \frac{\partial u_{\gamma}^{*}}{\partial z}+2 \widehat{A}_{\alpha 333} \frac{\partial u_{3}^{*}}{\partial z}\right\}=\widehat{f}_{\alpha},(\alpha=1,2) \\
-\frac{\partial}{\partial z}\left\{2 \widehat{A}_{33 \alpha 3} \frac{\partial u_{\alpha}^{*}}{\partial z}+\widehat{A}_{3333} \frac{\partial u_{3}^{*}}{\partial z}\right\}=\widehat{f}_{3}
\end{array}\right\} \text { inL }^{2}(\Omega) .
$$

Proof. As (23) can be written,

$$
\begin{gathered}
\widehat{a}\left(\widehat{u}^{\varepsilon}, \widehat{\phi}\right)+\int_{\omega} \hat{k}|\widehat{\phi}-s| d x-\sum_{i=1}^{3} \int_{\Omega} \widehat{f}_{i}\left(\widehat{\phi}_{i}-\widehat{u}_{i}^{\varepsilon}\right) d x d z \\
\geq \widehat{a}\left(\widehat{u}^{\varepsilon}, \widehat{u}^{\varepsilon}\right)+\int_{\omega} \widehat{k}\left|\widehat{u}^{\varepsilon}-s\right| d x .
\end{gathered}
$$

Since the form $\widehat{a}(.,$.$) is a symmetry and K$-elliptic, and the fact that $\widehat{\phi} \longrightarrow \int_{\omega} \widehat{k}|\widehat{\phi}-s| d x$ is convex and lower semicontinuous, we deduce

$$
\begin{gathered}
\liminf _{\mathcal{\varepsilon} \longrightarrow 0}\left[\widehat{a}\left(\widehat{u}^{\varepsilon}, \widehat{u}^{\varepsilon}\right)+\int_{\omega} \widehat{k}\left|\widehat{u}^{\varepsilon}-s\right| d x\right] \\
\geq \widehat{a}\left(u^{*}, u^{*}\right)+\int_{\omega} \widehat{k}\left|u^{*}-s\right| d x
\end{gathered}
$$

Using Lemma 5, we let $\varepsilon$ tend to 0 in (47), to obtain

$$
\begin{aligned}
& 4 \int_{\Omega} \widehat{A}_{\alpha 3 \gamma 3} \frac{\partial u_{\gamma}^{*}}{\partial z} \frac{\partial}{\partial z}\left(\widehat{\phi}_{\alpha}-u_{\alpha}^{*}\right) d x d z \\
& \quad+2 \int_{\Omega} \widehat{A}_{\alpha 333} \frac{\partial u_{3}^{*}}{\partial z} \frac{\partial}{\partial z}\left(\widehat{\phi}_{\alpha}-u_{\alpha}^{*}\right) d x d z \\
& \quad+2 \int_{\Omega} \widehat{A}_{33 \alpha 3} \frac{\partial u_{\alpha}^{*}}{\partial z} \frac{\partial}{\partial z}\left(\widehat{\phi}_{3}-u_{3}^{*}\right) d x d z \\
& \quad+\int_{\Omega} \widehat{A}_{3333} \frac{\partial u_{3}^{*}}{\partial z} \frac{\partial}{\partial z}\left(\widehat{\phi}_{3}-u_{3}^{*}\right) d x d z \\
& \quad+\int_{\omega} \widehat{k}\left(|\widehat{\phi}-s|-\left|u^{*}-s\right|\right) d x \\
& \geq \sum_{i=1}^{3} \int_{\Omega} \widehat{f}_{i}\left(\widehat{\phi}_{i}-u_{i}^{*}\right) d x d z .
\end{aligned}
$$

This completes the proof of (44) if we cross (49) in the matrix form $A^{*}$. We choose in the variational inequation (49) $\widehat{\phi}_{i}=u_{i}^{*} \pm \psi_{i}$, where $\psi_{i} \in H_{0}^{1}(\Omega)$ (for $i=1,2,3$ ), and using Green's formula, we find

$$
\begin{aligned}
& -\int_{\Omega} \frac{\partial}{\partial z}\left\{4 \widehat{A}_{\alpha 3 \gamma 3} \frac{\partial u_{\gamma}^{*}}{\partial z}+2 \widehat{A}_{\alpha 333} \frac{\partial u_{3}^{*}}{\partial z}\right\} \psi_{\alpha} d x d z \\
& \quad-\int_{\Omega} \frac{\partial}{\partial z}\left\{2 \widehat{A}_{33 \alpha 3} \frac{\partial u_{\alpha}^{*}}{\partial z}+\widehat{A}_{3333} \frac{\partial u_{3}^{*}}{\partial z}\right\} \psi_{3} d x d z \\
& =\sum_{i=1}^{3} \int_{\Omega} \widehat{f}_{i} \psi_{i} d x d z,
\end{aligned}
$$

choosing $\psi_{3}=0$ and $\psi_{\alpha} \in H_{0}^{1}(\Omega)$; then, $\psi_{\alpha}=0$ and $\psi_{3} \epsilon$ $H_{0}^{1}(\Omega)$, we get (59).

Theorem 7. Under the assumptions of Theorem 6 then, the solution of the limit problem (44)-(46) is unique in $V_{z}$.

Proof. Suppose that there exists two solutions $u^{*}$ and $v^{*}$ of the variational inequality (44), and taking $\widehat{\phi}=v^{*}$ in (44), then $\widehat{\phi}=u^{*}$ in the inequality relating to $v^{*}$. By subtracting the two obtained inequalities, we have

$$
\left\langle A^{*} \cdot \frac{\partial}{\partial z}\left(v^{*}-u^{*}\right), \frac{\partial}{\partial z}\left(v^{*}-u^{*}\right)\right\rangle_{L^{2}(\Omega)} \leq 0 .
$$

We must now check that $A^{*}$ is ellipticity. So, we return to the properties of $\widehat{A}$ mentioned in (14) and (15); in particular, we choose symmetric tensors $\zeta$ that are given by $\xi_{\alpha \beta}=0$ (for $\alpha, \beta=1,2)$; otherwise, the rest of the components $\left(\xi_{i 3}\right)$ let it be whatever. Putting $\eta_{i}=\xi_{i 3}$, for $i=1,2,3$, we will get 


$$
\begin{aligned}
\widehat{A}_{i j k l} \xi_{k l} \xi_{i j}= & 4 \widehat{A}_{\alpha 3 \beta 3} \eta_{\beta} \eta_{\alpha}+2 \widehat{A}_{\alpha 333} \eta_{3} \eta_{\alpha} \\
& +2 \widehat{A}_{33 \alpha 3} \eta_{\alpha} \eta_{3}+\widehat{A}_{3333} \eta_{3} \eta_{3}=A_{i j}^{*} \eta_{j} \eta_{i} .
\end{aligned}
$$

Consequently, and as $|\xi|^{2} \geq|\eta|^{2}$, there exists a positive constant $\mu$, and for all vectors $\eta$ in $\mathbb{R}^{3}$, we have

$$
A_{i j}^{*} \eta_{j} \eta_{i} \geq \mu|\eta|^{2} .
$$

So, $A^{*}$ is ellipticity. Thus, the relation (44) implies that

$$
\mu\left\|\frac{\partial}{\partial z}\left(v^{*}-u^{*}\right)\right\|_{L^{2}(\Omega)}^{2} \leq 0 .
$$

Using Poincaré's inequality, we obtain

$$
\left\|v^{*}-u^{*}\right\|_{L^{2}(\Omega)}^{2} \leq\left(h_{\max }\right)^{2}\left\|\frac{\partial}{\partial z}\left(v^{*}-u^{*}\right)\right\|_{L^{2}(\Omega)}^{2}=0
$$

and the proof of uniqueness of $u^{*}$ is complete.

Theorem 8. Under the assumptions of Theorem 7, the traces $\left(s^{*}, \pi^{*}\right)$ with $s^{*}=\left(s_{i}^{*}\right)_{1 \leq i \leq 3}$ and $\pi^{*}=\left(\pi_{i}^{*}\right)_{1 \leq i \leq 3}$ defined by

$$
\begin{aligned}
s_{i}^{*}(x) & =u_{i}^{*}(x, 0), \\
\pi_{\alpha}^{*}(x) & =\left[4 \widehat{A}_{\alpha 3 \gamma 3} \frac{\partial u_{\gamma}^{*}}{\partial z}+2 \widehat{A}_{\alpha 333} \frac{\partial u_{3}^{*}}{\partial z}\right](x, 0) ; \pi_{3}^{*}(x) \\
& =\left[2 \widehat{A}_{33 \gamma 3} \frac{\partial u_{\gamma}^{*}}{\partial z}+\widehat{A}_{3333} \frac{\partial u_{3}^{*}}{\partial z}\right](x, 0),
\end{aligned}
$$

satisfy the following limit form of the Tresca boundary conditions:

$$
\left.\begin{array}{c}
\int_{\omega} \widehat{k}\left|\psi+s^{*}-s\right|-\left|s^{*}-s\right| d x-\int_{\omega} \pi^{*} \cdot \psi d x \geq 0 \forall \psi \in L^{2}(\omega)^{3}, \\
\left|\pi^{*}\right|<\widehat{k} \Rightarrow s^{*}=s \\
\left|\pi^{*}\right|=\widehat{k} \Rightarrow \exists \lambda>\text { 0suchthat } s^{*}=s+\lambda \pi^{*}
\end{array}\right\} \text { a.e.in } \omega .
$$

Moreover, if the coefficients $\widehat{A}_{i 3 j 3}$ for $1 \leq i, j \leq 3$, depending only on the variable $x$, we have the following weak form of the Reynolds equation:

$$
\int_{\omega}\left(\tilde{F}-\frac{h}{2} s^{*}+\int_{0}^{h} u^{*}(x, z) d z\right) \cdot \nabla \psi(x) d x=0, \forall \psi \in H^{1}(\omega),
$$

where $\operatorname{Inv} A^{*}(x)$ denotes the inverse of $A^{*}(x)$ and

$$
\tilde{F}(x)=\int_{0}^{h} \tilde{F}(x, \rho) d \rho-\frac{h}{2} \tilde{F}(x, h),
$$

$$
\tilde{F}(x, \rho)=\operatorname{Inv} A^{*}(x) \cdot \int_{0}^{\rho} \int_{0}^{\theta} \widehat{f}(x, y) d y d \theta .
$$

Proof. We now choose in the variational inequality (49) $\widehat{\phi}_{i}$ $=u_{i}^{*}+\psi_{i}$, where $\psi_{i} \in H_{\Gamma_{1} \cup \Gamma_{L}}^{1}(\Omega)$ for $i=1,2,3$, and then using Green's formula, we obtain

$$
\begin{aligned}
& -\int_{\Omega} \frac{\partial}{\partial z}\left\{4 \widehat{A}_{\alpha 3 \gamma 3} \frac{\partial u_{\gamma}^{*}}{\partial z}+2 \widehat{A}_{\alpha 333} \frac{\partial u_{3}^{*}}{\partial z}\right\} \psi_{\alpha} d x d z \\
& -\int_{\omega}\left(4 \widehat{A}_{\alpha 3 \gamma 3} \frac{\partial u_{\gamma}^{*}}{\partial z}+2 \widehat{A}_{\alpha 333} \frac{\partial u_{3}^{*}}{\partial z}\right) \psi_{\alpha}(x, 0) d x \\
& \quad-\int_{\Omega} \frac{\partial}{\partial z}\left\{2 \widehat{A}_{33 \gamma 3} \frac{\partial u_{\gamma}^{*}}{\partial z}+\widehat{A}_{3333} \frac{\partial u_{3}^{*}}{\partial z}\right\} \psi_{3} d x d z \\
& \quad-\int_{\omega}\left(2 \widehat{A}_{33 \gamma 3} \frac{\partial u_{\gamma}^{*}}{\partial z}+\widehat{A}_{3333} \frac{\partial u_{3}^{*}}{\partial z}\right) \psi_{3}(x, 0) d x \\
& \quad+\int_{\omega} \widehat{k}\left(\left|\psi+s^{*}-s\right|-\left|u^{*}-s\right|\right) d x \\
& \geq \sum_{i=1}^{3} \int_{\Omega} \widehat{f}_{i} \psi_{i} d x d z .
\end{aligned}
$$

On the other hand, from (46), we have

$$
\int_{\omega} \widehat{k}\left(\left|\psi+s^{*}-s\right|-\left|s^{*}-s\right|\right) d x-\int_{\omega}\left(\sum_{\alpha} \pi_{\alpha}^{*} \psi_{\alpha}+\pi_{3}^{*} \psi_{3}\right) d x \geq 0
$$

By density theorems, we find (57). For (58), we use the analogue of [10].

To prove (59), we use those similar steps as in $[2,5,9-$ 11 ], by integrating (46) from 0 to $z$, and taking into account $\widehat{A}_{i 3 j 3}$ depending only on $x$, we obtain

$$
\left\{\begin{array}{c}
-\left[4 \widehat{A}_{\alpha 3 \gamma 3}(x) \frac{\partial u_{\gamma}^{*}}{\partial z}(x, z)+2 \widehat{A}_{\alpha 333}(x) \frac{\partial u_{3}^{*}}{\partial z}(x, z)\right]+\pi_{\alpha}^{*}(x)=\int_{0}^{z} \widehat{f}_{\alpha}(x, y) d y, \\
-\left[2 \widehat{A}_{33 \gamma 3}(x) \frac{\partial u_{\gamma}^{*}}{\partial z}(x, z)+\widehat{A}_{3333}(x) \frac{\partial u_{3}^{*}}{\partial z}(x, z)\right]+\pi_{3}^{*}(x)=\int_{0}^{z} \widehat{f}_{3}(x, y) d y \\
-A^{*}(x) \cdot \frac{\partial u^{*}}{\partial z}+\pi^{*}(x)=\int_{0}^{z} \widehat{f}(x, y) d y .
\end{array}\right.
$$


It follows from (51) that it is a invertible matrix $A^{*}(x)$, for almost every $x \in \omega$. Therefore,

$$
-\frac{\partial u^{*}}{\partial z}+\operatorname{Inv} A^{*}(x) \cdot \pi^{*}(x)=\operatorname{Inv} A^{*}(x) \cdot \int_{0}^{z} \widehat{f}(x, y) d y
$$

By integrating between 0 and $z$, we obtain

$$
-u^{*}(x, z)+s^{*}(x)+z \operatorname{Inv} A^{*}(x) \cdot \pi^{*}(x)=\tilde{F}(x, z) .
$$

As $u_{i}^{*}(x, h(x))=0$, we have

$$
s^{*}(x)+h(x) \operatorname{Inv} A^{*}(x) \cdot \pi^{*}(x)=\int_{0}^{h(x)} \tilde{F}(x, y) d y .
$$

We integrate (67) from 0 to $h(x)$, and we obtain

$$
\begin{aligned}
& -\int_{0}^{h(x)} u^{*}(x, z) d z+h s^{*}(x)+\frac{h(x)^{2}}{2} \operatorname{Inv} A^{*}(x) \cdot \pi^{*}(x) \\
& \quad=\int_{0}^{h(x)} \tilde{F}(x, y) d y
\end{aligned}
$$

and by (68), we deduce that

$$
-\int_{0}^{h(x)} u^{*}(x, z) d z+\frac{h(x)}{2} s^{*}(x)-\tilde{F}(x)=0,
$$

such that $\tilde{F}$ is already defined in (61), and let us finally get the weak form (59) after multiplying (70) by $\nabla \psi(x)$ and integrate it in $\omega$.

\section{Conclusions}

We were able to find a framework to conclude that solving our original problem leads to solving a well-defined problem as in (44),(46) and (57)-(59) for the "small" parameter $\varepsilon$.

The key of the problem lies in the relation between the matrices $A^{\varepsilon}$ and $A^{*}$. Note that they have the same properties despite the difference in dimensions, therefore it played a key role in the transition from $u^{\varepsilon}$ to $u^{*}$.

Indeed, the special case

$$
A_{i j k l}^{\varepsilon}=\mu^{\varepsilon}\left(\delta_{i k} \delta_{j l}+\delta_{i l} \delta_{j k}\right)+\lambda^{\varepsilon} \delta_{i j} \delta_{k l}
$$

where $\lambda^{\varepsilon}, \mu^{\varepsilon}>0$ are the Lamé coefficients (see [13] pp. 102103) corresponds to the homogeneous and isotropic case of elastic materials, and has been studied in $[2,3,5]$. Thus also, the Stokes flow in [11] can be recovered when $\lambda^{\varepsilon}$ tends to 0 .

\section{Data Availability}

No data were used.

\section{Conflicts of Interest}

The authors declare that they have no conflicts of interest.

\section{Acknowledgments}

The authors would like to thank Professor A. Sili for suggesting the problem addressed in this work.

\section{References}

[1] G. Bayada and K. Lhalouani, "Asymptotic and numerical analysis for unilateral contact problem with Coulomb's friction between an elastic body and a thin elastic soft layer," Asymptotic Analysis, vol. 25, pp. 329-362, 2001.

[2] H. Benseridi and M. Dilmi, "Some inequalities and asymptotic behavior of a dynamic problem of linear elasticity," Georgian Mathematical Journal, vol. 20, no. 1, pp. 25-41, 2013.

[3] D. Benterki, H. Benseridi, and M. Dilmi, "Asymptotic study of a boundary value problem governed by the elasticity operator with nonlinear term," Adv. Appl. Math. Mech., vol. 6, no. 2, pp. 191-202, 2014.

[4] H. Irago, J. M. Viaño, and Á. Rodríguez-Arós, “Asymptotic derivation of frictionless contact models for elastic rods on a foundation with normal compliance," Nonlinear Analysis: Real World Applications, vol. 14, no. 1, pp. 852-866, 2013.

[5] Y. Letoufa, H. Benseridi, and M. Dilmi, "Asymptotic study of a frictionless contact problem between two elastic bodies," Journal of Mathematics and Computer Science, vol. 16, no. 3, pp. 336-350, 2016.

[6] R. Monneau, F. Murat, and A. Sili, Error Estimate for the Transition 3d-1d in Anisotropic Heterogeneous Linearized Elasticity, 2002, Preprint.

[7] F. Murat and A. Sili, "Asymptotic behavior of solutions of the anisotropic heterogeneous linearized elasticity system in thin cylinders," Comptes Rendus de l'Académie des Sciences - Series I- Mathematics, vol. 328, no. 2, pp. 179-184, 1999.

[8] J. M. Viaño, Á. Rodríguez-Arós, and M. Sofonea, “Asymptotic derivation of quasistatic frictional contact models with wear for elastic rods," Journal of Mathematical Analysis and Applications, vol. 401, no. 2, pp. 641-653, 2013.

[9] H. Benseridi, Y. Letoufa, and M. Dilmi, "On the asymptotic behavior of an interface problem in a thin domain," M. Proc. Natl. Acad. Sci., India, Sect. A Phys. Sci., vol. 89, no. 2, pp. 110, 2019.

[10] M. Boukrouche and R. el Mir, "Asymptotic analysis of a nonNewtonian fluid in a thin domain with Tresca law," Nonlinear Analysis: Theory Methods \& Applications, vol. 59, no. 1-2, pp. 85-105, 2004.

[11] D. Benterki, H. Benseridi, and M. Dilmi, “Asymptotic behavior of solutions to a boundary value problem with mixed boundary conditions and friction law," Boundary Value Problems, vol. 2017, no. 1, 2017.

[12] S. Manaa, H. Benseridi, and M. Dilmi, “3D-2D asymptotic analysis of an interface problem with a dissipative term in a dynamic regime," Boletín de la Sociedad Matemática Mexicana, vol. 27, no. 1, p. 10, 2021.

[13] G. Duvant and J. L. Lions, Les Inéquations en Mécanique et en physique, Dunod, Paris, 1972.

[14] C. Baiocchi and A. Capelo, "Variational and quasivariational inequalities," in Applications to Free-Boundary Problems, John Wiley, Chichester, 1984.

[15] N. Bellomo and L. de Socio, "Initial/boundary value problems for the semidiscrete Boltzmann equation: Analysis by 
Adomian's decomposition method," Journal of Mathematical Analysis and Applications, vol. 128, no. 1, pp. 112-124, 1987.

[16] J. Necas and I. Hlavacek, Mathematical theory of elastic and elastico plastic bodies: an introduction, Elsevier Scientific Publishing Company, Amsterdam, Oxford, New York, 1981.

[17] I. Ekeland and R. Temam, Analyse Convexe et Problèmes Variationnels, Dunod, Paris, 1974.

[18] N. Kikuchi and J. T. Oden, Contact problems in elasticity, a study of variational inequalities and finite element methods, SIAM, Philadelphia, 1988.

[19] M. Sofonea and A. Matei, Mathematical Models in Contact Mechanics, London Math. Soc, Lecture Note Ser., Cambridge University Press, Cambridge, 2012.

[20] V. Girault and P. A. Raviart, Finite element approximation of the NavierStokes equations, Springer-Verlag, 1979. 